\title{
A 2D scintillator-based proton detector for high repetition rate experiments
}

\author{
M. Huault ${ }^{1,2}$, D. De Luis ${ }^{1}$, J. I. Apiñaniz ${ }^{1}$, M. De Marco ${ }^{1}$, C. Salgado ${ }^{1,2}$, N. Gordillo ${ }^{3,4}$, \\ C. Gutiérrez Neira ${ }^{3}$, J. A. Pérez-Hernández ${ }^{1}$, R. Fedosejevs ${ }^{5}$, G. Gatti ${ }^{1}$, L. Roso ${ }^{1,2}$, and L. Volpe ${ }^{1,6}$ \\ ${ }^{1}$ Centro de Laseres Pulsados, Building M5, Science Park, Calle Adaja 8, 37185 Villamayor, Salamanca, Spain \\ ${ }^{2}$ Universidad de Salamanca, Patio de Escuelas 1, 37008 Salamanca, Spain \\ ${ }^{3}$ CMAM, Universidad Autónoma de Madrid, Campus de Cantoblanco, E-28049 Madrid, Spain \\ ${ }^{4}$ Grupo de Electrónica y Semiconductores, Departamento de Física Aplicada, Universidad Autónoma de Madrid, Cantoblanco, \\ E-28049 Madrid, Spain \\ ${ }^{5}$ University of Alberta, 116 St85 Ave, Edmonton, AB T6G 2R3, Alberta, Canada \\ ${ }^{6}$ Laser-Plasma Chair at the University of Salamanca, Patio de Escuelas 1, Salamanca, Spain \\ (Received 30 April 2019; revised 3 June 2019; accepted 2 September 2019)
}

\begin{abstract}
We present a scintillator-based detector able to measure the proton energy and the spatial distribution with a relatively simple design. It has been designed and built at the Spanish Center for Pulsed Lasers (CLPU) in Salamanca and tested in the proton accelerator at the Centro de Micro-Análisis de Materiales (CMAM) in Madrid. The detector is capable of being set in the high repetition rate (HRR) mode and reproduces the performance of the radiochromic film detector. It represents a new class of online detectors for laser-plasma physics experiments in the newly emerging high power laser laboratories working at HRR.
\end{abstract}

Keywords: high repetition rate; laser particle acceleration; online detector; proton diagnostic; scintillator

\section{State of the art}

The advent of high power lasers (HPL) working at high repetition rate (HRR) is nowadays a reality and HRR proton sources are now routinely produced with energies ranging from a few to tens of MeV. Laser-driven proton sources are characterized by a divergence that in several measurements has been proved to be related to the energy of the protons and the spatial distribution of the proton beam ${ }^{[1]}$.

Laser-driven proton beams are becoming more and more important for applications in different fields of physics ${ }^{[2]}$, chemistry and materials science ${ }^{[3]}$, as well as biology, medicine $^{[4]}$ and cultural heritage ${ }^{[5]}$. For this, spatial and energy characterization of the proton beams nowadays plays an important role for the potential use of such sources. The first demonstration of laser-driven proton production was carried out in laser systems working in the single-shot mode, and one of the most used diagnostic consists of a series of radiochromic films $(\mathrm{RCFs})^{[6]}$ placed one after the other

Correspondence to: L. Volpe, Centro de Láseres Pulsados (CLPU), Edificio M5, Parque Científico. C/Adaja 8, 37185 Villamayor, Salamanca, Spain.Email:1volpe@clpu.es

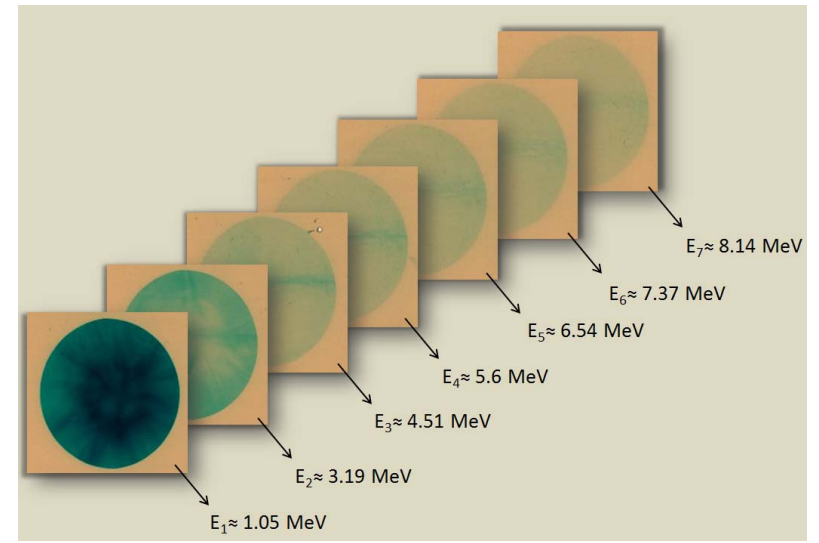

Figure 1. Sample of Gafchromic HD-V2 radiochromic films irradiated by protons at the Spanish Center for Pulsed Laser (CLPU).

and able to recover the spatial distribution as a function of the proton energy ${ }^{[7]}$. Figure 1 shows an example of the radiochromic stack used at CLPU to characterize the protons generated by the interaction of the laser VEGA $2(800 \mathrm{~nm}$, $30 \mathrm{fs}, 200 \mathrm{TW}, 6 \mathrm{~J})$ with a $6 \mu \mathrm{m}$-thick aluminium target ${ }^{[8]}$. 
The possibility of extending this technique to the HRR mode of operation is nowadays a challenge in the laserplasma community, and several laboratories and research groups are working on this. The main idea is to substitute the active RCF layers with scintillator detectors capable of transforming the ion energy deposition into light that can then be collected by an optical CCD camera. Several research groups have proposed special online configurations to imitate the RCF stack but, up to now, only a partial extension of the RCF capabilities was possible. During 2011 and 2012, two research groups from the United Kingdom and from Germany proposed scintillator-based detectors. The group from Rutherford Appleton Laboratory (RAL) ${ }^{[9]}$ proposed using a detector sensitive to three different wavelengths. The group from Dresden ${ }^{[10]}$ proposed a stack of scintillators placed one after the other, as in the RCF stack, with a readout system looking at the transversal scintillation emission. Such devices have been tested in a proton beam accelerator and are currently used in the Dresden laboratory. Both detectors can partially reproduce the working mode of the RCF stacks even though they increase the complexity of the viewing system and the data interpretation.

\section{Detector design}

We present a scintillator-based detector able to measure both the proton energy and its transversal spatial distribution along the propagation axis and of being set at HRR. It consists of a series of scintillators placed similarly to an RCF stack (shown in Figure 1), but positioned with a relative angle with respect to one to another in order to leave a free field of view for an imaging system looking at the back side of each layer. The imaging system can be arranged depending on the spatial condition and is not a critical part of the device. Each scintillator plate is covered on the front side by an aluminium foil to protect it from the light emission produced by the previous scintillator plate. The relative angle $\phi$ between each layer is the key factor in the design because it permits the acquisition of the full 2D proton distribution for each of the layers composing the stack. It is a relevant parameter because the total size of the detector depends critically on it, since increasing the angle increases the total length of the detector and also the size of the detector elements must be increased to detect a given proton emission solid angle at a farther distance from the source.

To assess the system in detail we assume a proton beam propagating in a symmetric cone emission with half-angle $\theta$ (see Figure 2), where the transversal $(D)$ and longitudinal $(d)$ dimension of each scintillator plate (which are assumed to be equal in size) perpendicular to the proton beam direction can be written as

$$
D=L \cos (\phi) ; \quad d=L \sin (\phi)
$$

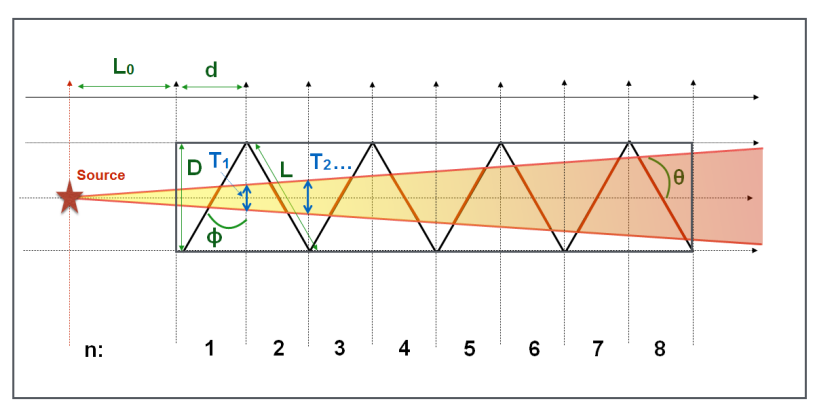

Figure 2. $2 \mathrm{D}$ top view of detector; the proton beam solid angle is parametrized by the internal half-angle $\theta$, the detector dimension $D$ is represented by the length of the scintillator plate $L$ and the relative halfangle between the plates $\phi$, and $n$ is the number of layers. $L_{0}$ is the distance between the proton source and the detector, $d$ is the longitudinal dimension of the scintillator plate, and $T_{1}, \ldots, T_{n}$ represent the projections of the proton beam solid angle for each plate.

The projection of the proton emission cone in the scintillator plate can be written as

$$
\begin{gathered}
T_{n}(\theta, \phi)=2 L_{n}(\phi) \tan (\theta), \\
L_{n}(\phi)=L_{0}+n L \sin (\phi) .
\end{gathered}
$$

$L_{n}$ is the effective length of the detector considered from the proton source emission. Let us note that $L_{0}$ must be greater than zero because a minimum distance between the detector and the source must be allowed to accommodate a magnet to deflect the electrons generated in the interaction process so as not to affect the scintillation signal. Finally, $L_{n}$ depends on the angle between two successive plates $\phi$, the dimension $L$ and the number $n$ of scintillator foils.

The working condition can be written as

$$
D>T_{n}(\theta, \phi)
$$

where the size of the transverse projection of the scintillator $D$ must be larger than the projection of the proton solid angle $T_{n}$. This can be solved as

$$
\begin{gathered}
n<n_{0}(\theta, \phi)+n^{\prime}\left(L_{0}, L, \phi\right), \\
n_{0}(\theta, \phi)=\frac{1}{2 \tan (\theta \tan \phi)}, \\
n^{\prime}\left(L_{0}, L, \phi\right)=-\frac{L_{0}}{L} \frac{1}{\sin \phi},
\end{gathered}
$$

where $n_{0}=n\left(\theta, \phi, L_{0}=0, L\right)$.

\subsection{Case $L_{0}=0$}

The case $L_{0}=0$ corresponds to the assumption that the proton source is just placed at the surface corresponding to 


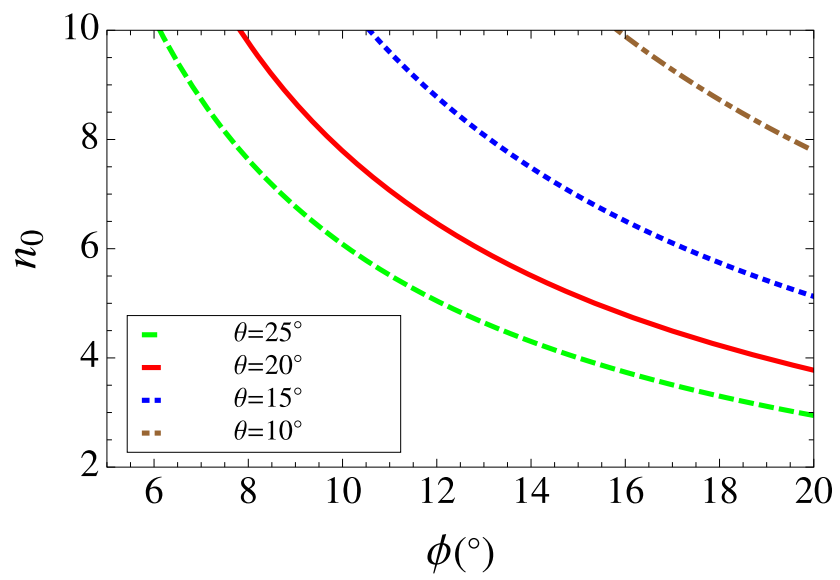

Figure 3. Case $L_{0}=0$. Plot of the number of layers $n_{0}$ versus the half-angle between the plates $\phi$ (according to Equation (5)) for different divergence half-angles $\theta$. When the curves $n_{0}(\phi)$ are above a given fixed $n_{0}$ value, the design of the detector is such that proton energies corresponding to the $n_{0}$ value are detectable. As an example, for a proton beam with a $40^{\circ}$ divergence $\left(\theta=20^{\circ}\right)$, six layers can work with a maximum angle $\phi \sim 13^{\circ}$, while for eight layers $\phi \sim 10^{\circ}$. It is important to note that the proton energy corresponding to the $n$th layer depends on the thickness and composition of the layer.

$n=0$, so the system becomes

$$
n<n_{0}(\theta, \phi) \text {. }
$$

Equation (5) can be studied as a function of $\phi$ (for a given value of $\theta$; here 25, 20, 15 and 10 degrees).

Figure 3 shows the number of layers $n_{0}$ (representing Equation (5)) as a function of $\phi$ for different divergence halfangles $\theta$. The relative angle between the layers $(\phi)$ needs to be reduced as much as possible to maximize the possible number of layers while maintaining a reasonable dimension of the detector. As an example, the spatial resolution of our imaging system $\left(\phi=12.5^{\circ}\right)$ is about $58 \mu \mathrm{m}$ in the vertical axis and $100 \mu \mathrm{m}$ in the horizontal axis, with a contraction factor of $\sim 1.7$ in the horizontal axis. The relative angle $\phi$ should not be below $10^{\circ}$ when considering a reasonable spatial resolution of the imaging system.

\subsection{Case $L_{0} \neq 0$}

Assuming a proton divergence with an half-angle $\theta=20^{\circ}$ and the relative half-angle between two plates $\phi=12^{\circ}$, we can represent $n$ (see Equation (4)) as a function of $L_{n}$ for different values of $L_{0}$, as shown in Figure 4.

The result is that increasing $L_{0}$ proportionally increases $L$, and of course the total longitudinal dimension $L_{n}$. These examples show how the energy range of the detector is strongly related to the geometric parameters. Such limitations can easily be overcome with special, dedicated adjustments of the detector design and position in the interaction chamber. In addition, two other physical aspects need to be considered:

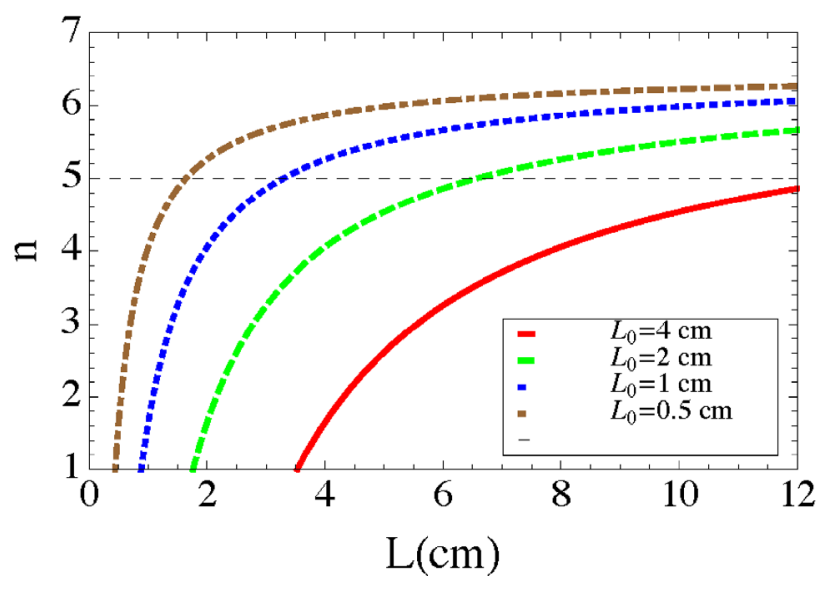

Figure 4. The number of scintillator layers $n$ is represented as a function of the scintillator foil size $(L)$ for different values of $L_{0}$ and for fixed values of $\phi=12^{\circ}$ and $\theta=20^{\circ}$. Different values of $L_{0}$ are plotted, representing the distance between the detector and the interaction point position. As an example (dashed line in the graph), a detector with $n=5$ layers needs to be built with a size $L$ greater than: $\sim 1.5 \mathrm{~cm}\left(L_{0}=0.5 \mathrm{~cm}\right) ; \sim 3.5 \mathrm{~cm}\left(L_{0}=\right.$ $1 \mathrm{~cm}), \sim 6.5 \mathrm{~cm}\left(L_{0}=2 \mathrm{~cm}\right) ; \sim 12 \mathrm{~cm}\left(L_{0}=4 \mathrm{~cm}\right)$.

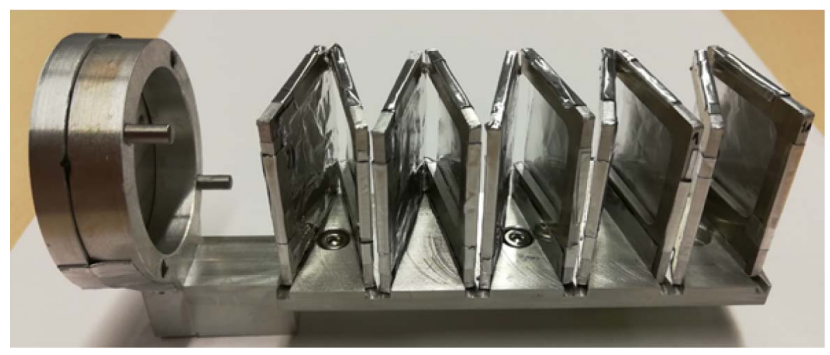

Figure 5. Lateral view of the detector with a longitudinal dimension of the base between the first and the last plate of approximately $90 \mathrm{~mm}\left(L_{10}=\right.$ $90 \mathrm{~mm}$ with $\left.L_{0}=0\right)$. The plates are separated from each other with a relative angle $\phi=12.5^{\circ}$ and have a dimension of $L=20 \mathrm{~mm}$.

(i) experimental data have shown that the proton divergence is reduced by increasing the proton energy; (ii) we have estimated the contribution of proton multiple scattering in blurring the signal in the rearmost layers to be in the range $2^{\circ}-3^{\circ}$ (half-angle). Both effects must be considered in order to properly design the detector.

\section{Implementation and preliminary calibration of the detector}

A first detector prototype has been designed and constructed at the Spanish Center for Pulsed Laser (CLPU) in Salamanca and tested at the Centro de Micro-Análisis de Materiales (CMAM) of the Universidad Autónoma de Madrid, where a collimated proton beam up to $10 \mathrm{MeV}$ is available for user access.

Figure 5 shows a customized version of the detector which was used in the accelerator in Madrid, with all the relevant parameters; the proton beam was $10 \mathrm{MeV}$ energy with 


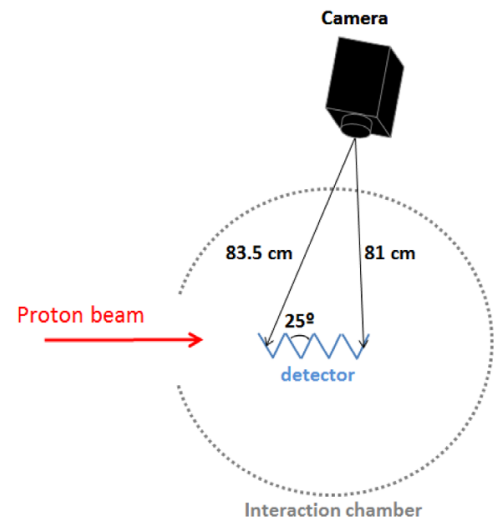

Figure 6. Top view of the interaction chamber with the detector placed inside and the camera set outside the chamber to record the signal.

Table 1. BC-400 scintillator main properties.

\begin{tabular}{lc}
\hline Base & Polyvinyltoluene \\
\hline Density & $\rho=1.032 \mathrm{~g} / \mathrm{cm}^{3}$ \\
Refractive index & $n=1.58$ \\
Softening point & $70^{\circ} \mathrm{C}$ \\
Light output (\% of anthracene) & $65 \%$ \\
Rise time & $0.9 \mathrm{~ns}$ \\
Decay time & $2.4 \mathrm{~ns}$ \\
Wavelength of maximum emission & $423 \mathrm{~nm}$ \\
Bulk light attenuation length & $250 \mathrm{~cm}$ \\
\hline
\end{tabular}

$\Delta E / E<1 \%$. In the case of a proton accelerator, due to the very low beam divergence, there are no constraints related to the possible number of layers. We implemented 10 equal thickness layers for the test in CMAM in order to resolve energies greater than the maximum $10 \mathrm{MeV}$ achievable in CMAM (in the case of a divergent beam, the design has to be done by first fixing the number of layers according to the beam divergence, then adding a stopping material in front of the detector in order to have the maximum possible proton energy in the last layer). In particular, the detector is made using 10 plates of the scintillator BC-400 (see Table 1 for properties) placed one after the other with an angle of $25^{\circ}$ between them $\left(\phi=12.5^{\circ}\right)$. Each plate is about $150 \mu \mathrm{m} \pm$ $50 \mu \mathrm{m}$ thick with a free detector area of $20 \mathrm{~mm} \times 20 \mathrm{~mm}$. $10 \mu \mathrm{m}$-thick aluminium foil is placed on the front side of each scintillator plate to protect it from the light emission produced by the previous scintillator plate.

The detector was placed in the middle of the interaction chamber, on the front part of a 4-axis goniometer, able to rotate $360^{\circ}$ around the propagation axis of the proton beam. The emission was collected from the rear side of each scintillator (downstream side) with a CCD camera (Point Grey Blackfly monochrome model; 1920 by 1200 pixels) and an objective (NIKON AF-S DX NIKKOR 18-105 mm $f / 3.5-5.6 \mathrm{G}$ ED VR) placed outside the chamber at about $83.5 \mathrm{~cm} \pm 0.5 \mathrm{~cm}$ from the first plate and $81 \mathrm{~cm} \pm 0.5 \mathrm{~cm}$ from the 10th plate (see Figure 6).

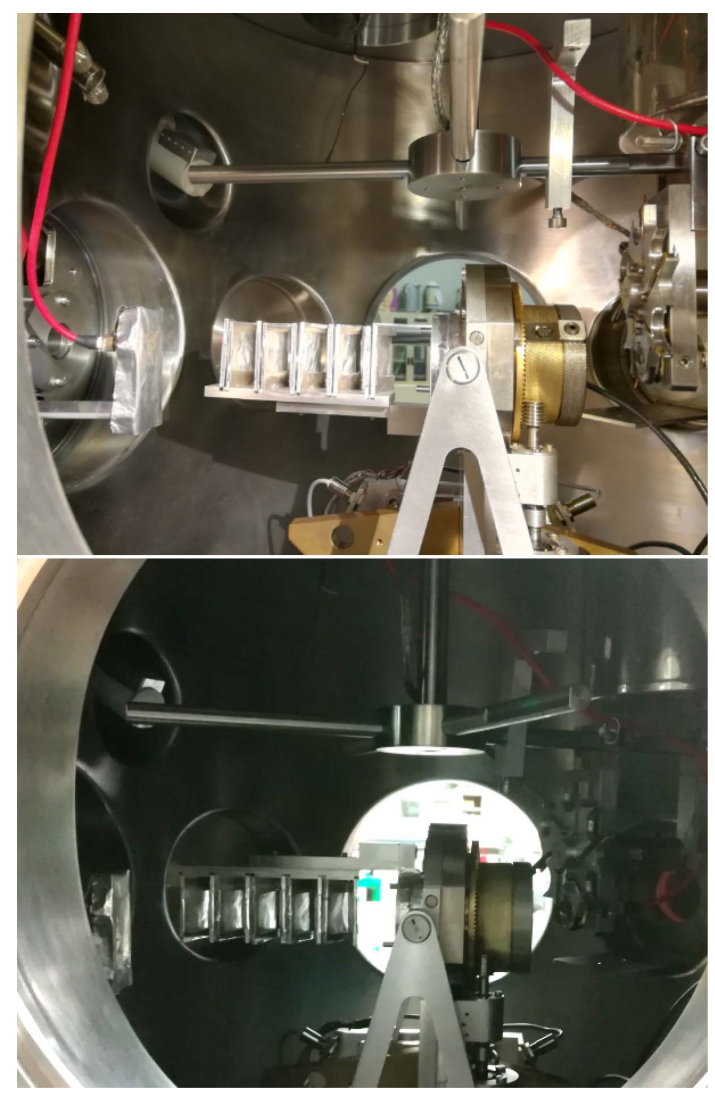

Figure 7. The top picture represents configuration 1, in which an odd number of scintillator plates are imaged by the camera. The bottom picture represents configuration 2 with the imaging of the pair scintillator plates.

The original design of the detector uses two cameras looking from opposite sides. Due to the experimental constraints and since the proton source was very stable, two configurations of irradiation were used to image the full detector with the same camera. The odd plates with the numbers 1, 3, 5, 7 and 9 were pictured when the goniometer was in the normal position (rotation axis at $0^{\circ}$ ) and the pair plates numbers $2,4,6,8$ and 10 when the goniometer was at $180^{\circ}$ of rotation (see Figure 7 ). The $10 \mathrm{MeV}$ proton beam irradiated the detector under a $10^{-6}$ mbar vacuum. Figure 8 represents a simulation of the energy deposited by a proton beam of $10 \mathrm{MeV}$ carried out using the FLUKA modelling code in order to reproduce the experimental results. It is expected to obtain the Bragg peak within the 6th plate and the aluminium filter of the 7 th plate.

Figure 9(a) shows the signal recorded during the two configurations of irradiation, which have been combined to give the signal in a single image. As an example the total deposited energy per proton for each scintillator plate has been extracted from the FLUKA simulation and is represented in Figure 9(b). It is in good agreement with the scintillator response in Figure 9(c). The light output has been obtained from recorded pictures by the conversion of 


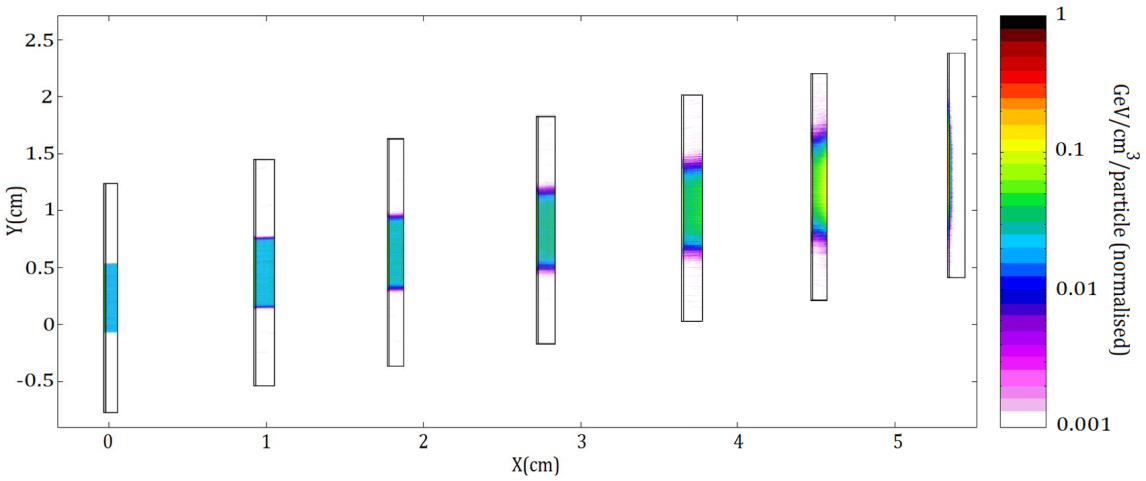

Figure 8. Top view of a Monte Carlo simulation (using the FLUKA code) of the proton energy deposited in the scintillator layers of the detector. The proton beam arrives with an incidence angle of $12.5^{\circ}$ on each plate. To facilitate the simulation procedure, the scintillator foils are placed parallel to each other; this configuration is totally equivalent to the original one and does not affect the general results. The colour scale represents the amount of energy lost by a $10 \mathrm{MeV}$ proton beam in each scintillator. The $x$ axis and $y$ axis represent the respective spatial distribution of the deposited energy (plate thicknesses are not shown to scale for easier visualization).

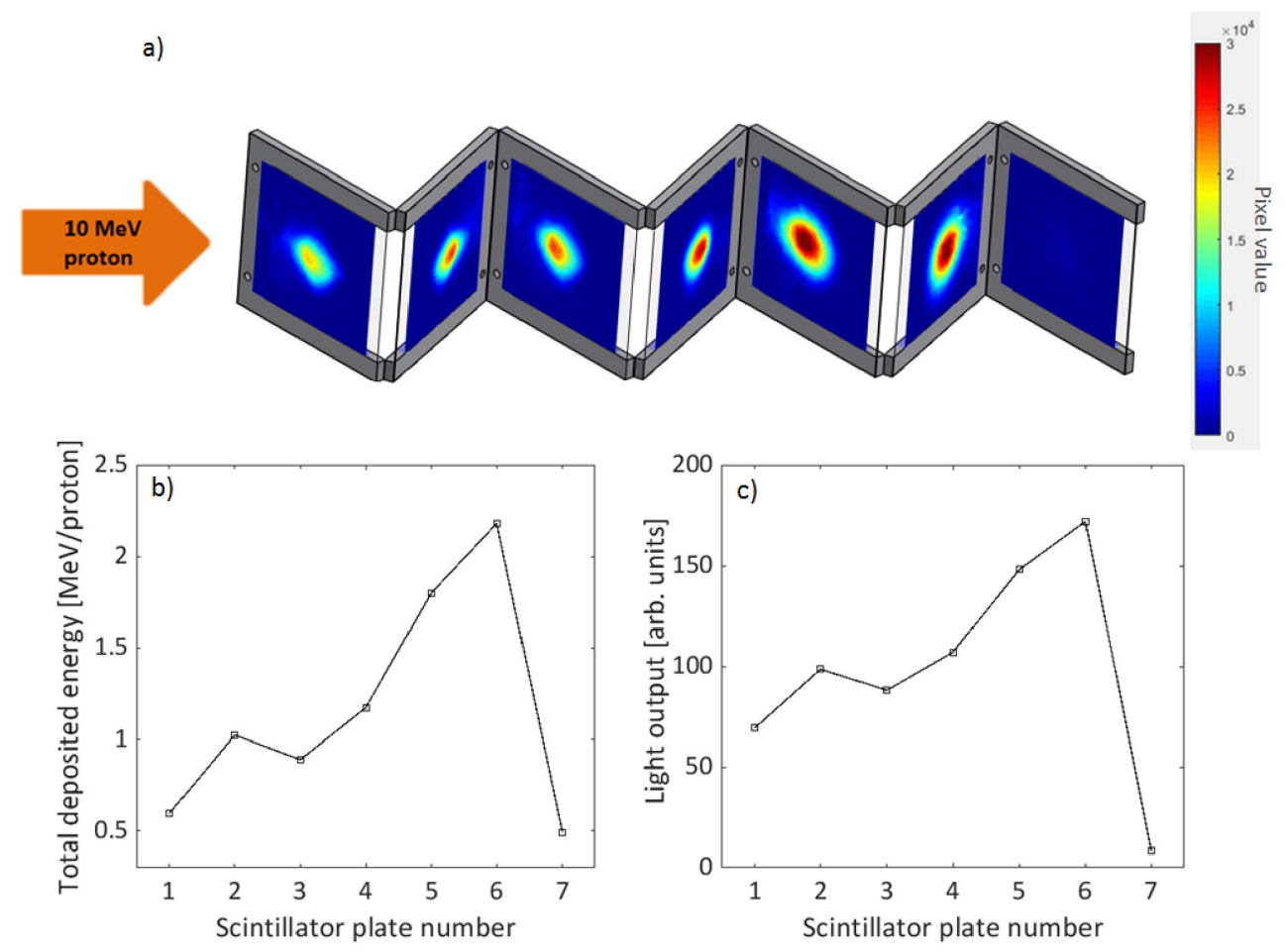

Figure 9. (a) Experimental signal obtained by the CCD camera during irradiation with a $10 \mathrm{MeV}$ proton beam, with the colour scale giving pixel values artificially overlaid on a 3D representation of the detector. (b) Example of Monte Carlo simulation (obtained with FLUKA) representing the transversally integrated deposited energy per particle for each scintillator plate irradiated by a $10 \mathrm{MeV}$ proton beam. (c) Response of the scintillator (light output) to $10 \mathrm{MeV}$ proton beam irradiation. We can observe a peak of energy in layer 2 (in panels (b) and (c)) due the thickness difference between layers 2 and 3 $(180 \mu \mathrm{m}$ against $140 \mu \mathrm{m})$. Then, far from the Bragg peak, a proton will deposit more energy in layer 2 than in layer 3. Each plate $(n)$ has a different thickness (due to uncontrollable variation during the fabrication process): $n_{1}=120 \mu \mathrm{m} ; n_{2}=180 \mu \mathrm{m} ; n_{3}=140 \mu \mathrm{m} ; n_{4}=160 \mu \mathrm{m} ; n_{5}=190 \mu \mathrm{m} ; n_{6}=130 \mu \mathrm{m}$; $n_{7}=150 \mu \mathrm{m}$ (lines in panels (b) and (c) are visual guides and not fits).

pixel values into photons. We can observe a slight flattening of the scintillator response around the Bragg peak (high stopping power) that can be interpreted as saturation of the response and is due to the quenching effect. A paper will be published describing the calibration in detail, where it will be shown that there is a linear response for proton flux but non- linearity in response to the absorbed dose for high stopping power proton deposition. Knowing the corrective factor of this effect ${ }^{[11]}$, the detector can be used for quantitative $2 \mathrm{D}$ spatial distribution measurements.

Considering a laser-ion acceleration experiment, the divergence of the particle beam has to be considered in the 
detector design. Indeed, increasing the total size of the detector will induce an increase of the final beam size, which, in combination with the multiple scattering, will entail a reduction of the spatial and energy resolutions. In addition, the last layers will receive a reduced number of protons per unit area, also reducing the detector sensitivity. The optimum detector design must be defined by prioritizing one parameter with respect to the other, even if a good general rule is to keep the length of the detector short to maintain a small beam size for a given number of layers. This can be done either by reducing the angle $\phi$ or reducing the lateral size $L$ of the scintillator foils.

\section{Discussion and conclusion}

A scintillator-based 2D ion detector for HRR experiments has been designed and built at CLPU, and tested using a proton accelerator at the CMAM in Madrid. The scintillator detector, to our knowledge, is a diagnostic device that looks very similar to an RCF stack diagnostic. Throughout the detailed analysis reported here, we have shown that it is possible to account for the laser-driven proton divergence by maintaining a compact size of detector. We have also shown that the detector can be implemented with an additional permanent magnet to remove most of the electron population, although the effect it has on the proton flux distribution at each energy will have to be mitigated.

Finally, the presented design of this 2D ion detector is promising for replacement of the classical RCF stack detector for the HRR mode of operation. It represents a new class of online detectors to support laser-plasma physics experiments in the newly emerging high power laser systems operating at HRR.

\section{Acknowledgements}

The authors acknowledge the FURIAM project FIS20134774-R, PALMA project FIS2016-81056-R, LaserLab Europe IV Grant No. 654148, Junta de Castilla y León Grant
No. CLP087U16 and Unidad de Investigación Consolidada (UIC) 167 from Junta de Castilla y León.

\section{References}

1. R. A. Snavely, M. H. Key, S. P. Hatchett, T. E. Cowan, M. Roth, T. W. Phillips, M. A. Stoyer, E. A. Henry, T. C. Sangster, M. S. Singh, S. C. Wilks, A. MacKinnon, A. Offenberger, D. M. Pennington, K. Yasuike, A. B. Langdon, B. F. Lasinski, J. Johnson, M. D. Perry, and E. M. Campbell, Phys. Rev. Lett. 85, 2945 (2000).

2. S. Fritzler, V. Malka, G. Grillon, J. P. Rousseau, F. Burgy, E. Lefebvre, E. dHumières, P. McKenna, and K. W. D. Ledingham, Appl. Phys. Lett. 83, 3039 (2003).

3. S. A. E. Johansson, Fresenius J. Anal. Chem. 324, 635 (1986).

4. V. Malka, S. Fritzler, E. Lefebvre, E. dHumières, R. Ferrand, G. Grillon, C. Albaret, S. Meyroneinc, J.-P. Chambaret, A. Antonetti, and D. Hulin, Med. Phys. 31, 1587 (2004).

5. M. Barberio, S. Veltri, M. Scisciò, and P. Antici, Sci. Rep. 7, 40415 (2017)

6. J. S. Green, A. P. L. Robinson, N. Booth, D. C. Carroll, R. J. Dance, R. J. Gray, D. A. MacLellan, P. McKenna, C. D. Murphy, D. Rusby, and L. Wilson, Appl. Phys. Lett. 104, 214101 (2014).

7. L. Volpe, R. Jafer, B. Vauzour, Ph. Nicolai, J. J. Santos, F. Dorchies, C. Fourment, S. Hulin, C. Regan, F. Perez, S. Baton, K. Lancaster, M. Galimberti, R. Heathcote, M. Tolley, Ch. Spindloe, W. Nazarov, P. Koester, L. Labate, L. A. Gizzi, C. Benedetti, A. Sgattoni, M. Richetta, J. Pasley, F. N. Beg, S. Chawla, D. P. Higginson, A. G. MacPhee, and D. Batani, Plasma Phys. Control. Fusion 53, 032003 (2011).

8. L. Volpe, R. Fedosejevs, G. Gatti, J. A. Pérez-Hernández, C. Méndez, J. Apiñaniz, X. Vaisseau, C. Salgado, M. Huault, S. Malko, G. Zeraouli, V. Ospina, A. Longman, D. De Luis, K. Li, O. Varela, E. García, I. Hernández, J. D. Pisonero, J. García Ajates, J. M. Alvarez, C. García, M. Rico, D. Arana, J. Hernández-Toro, and L. Roso, High Power Laser Sci. Eng. 7, e25 (2019).

9. J. S. Green, G. G. Scott, D. Neely, D. Kirby, D. Parker, M. J. Merchant, K. Kirkby, and S. Green, CLF Annual Report 2010 2011 (2011).

10. J. Metzkes, L. Karsch, S. D. Kraft, J. Pawelke, C. Richter, M. Schrer, M. Sobiella, N. Stiller, K. Zeil, and U. Schramm, Rev. Sci. Instrum. 83, 123301 (2012).

11. L. Torrisi, Nucl. Instrum. Methods Phys. Res. B 170, 523 (2000). 